\title{
COMENTARIO
}

\section{LA TEORIA DE LA CIENCIA COMO INVESTIGACIÓN Y REFLEXIÓN}

El hecho de que en las sociedades altamente industrializadas la ciencia desempeñe un papel cada vez mayor, por razón de la ciencialización brusca de la política y de amplios sectores de la práctica profesional, y pueda ser vista ya como la fuerza productiva decisiva, se ha convertido entretanto casi en un lugar común de la discusión sobre política. Se ha empezado a ver que, en la medida en que el progreso técnico científico acumula nuestra fuerza para disponer de la naturaleza y el hombre, va siendo cada vez más necesario analizar este potencial, es decir, investigar sus posibles efectos y resultados colaterales y manejarlo en forma responsable, es decir, transformarlo para el despliegue y mejoramiento libres de la vida humana en la práctica.

Si se omitieran ambas, tanto una teoría crítica como una praxis razonable para la dirección de esta segunda revolución científica industrial, se sobrepasaría con mucho el temor de Marx acerca del hechicero desatado de la fuerza productiva industrial frente a la fuerza productiva desencadenada técnico científica - por ejemplo, por un envenenamiento de la ecósfera, como resultado colateral material con el que no se contaba o también por una manipulación e incapacitación técnica totalitaria del hombre como resultado colateral político cultural del progreso. El que esta situación problemática se haya visto como desafío de la filosofía y teoría de la ciencia, es ante todo el beneficio de la "teoría crítica", a saber, de la Kritik der instrumentellen Vernunft de Horkheimer, de la Dialektik der Aufklärung de Horkheimer y Adorno, del One dimensional Man de Marcuse. A decir verdad, la teoría crítica no ha correspondido aún lo bastante a la situación pro. blemática actual, que acabamos de esbozar, y a su necesidad, tanto de la explicación del progreso científico como a criterios para su dirección responsable; lo que no le fue tampoco posible dentro de los límites de la filosofía social ideológico crítica y la crítica de la cultura, y sobre todo en el marco materialistamente limitado de su manera de ver. Sin embargo, creó, en unión con la orientación marxista por ella marcada o de otro tipo, una conciencia del problema, tanto en los movimientos revolucionarios de China y del Tercer Mundo, y determinado movimiento internacional estudiantil; conciencia ante la cual no pueden retrotraerse hacia la teoria pura, sin pérdida de prestigio, ni la metaciencia, ni la teoría de la ciencia y la filosofía. En el desarrollo posterior de la teoría crítica, o en todo caso fomentado por ella, en especial Habermas y Apel han transformado el pensamiento dialéctico hermenéutico en una teoría de la ciencia y una antropología del conocimiento, que vuelve a poner en el centro la dimensión pragmática de las ciencias y la pregunta acerca de su carácter ético práctico. Por parte de las escuelas analí. 
ticas ha surgido, en seguimiento crítico de Popper, un pragmatic turn, que ha tematizado el growth of knowledge y las revoluciones científicas (Kuhn, Lakatos) y ha desencadenado el desarrollo de una teoría de la investigación y una ciencia de la investigación (Törnebohm, Radnitzky). ${ }^{1}$

El principio que se acaba de mencionar, que es trabajado desde 1964 en el Institute for the Theory of Science de Goteborg, ${ }^{2}$ quiere mejorar la autocomprensión de la investigación por un control de resultados, de "modos de ver" y estrategias de investigación teoréticos, quiere ofrecer el mismo "modos de ver" críticos, que lleven a un nuevo standard $d^{3}$ nuestro conocimiento sobre el progreso de la ciencia, y quiere contribuir a la racionalización de la planeación de la investigación."

\section{El principio de Goteborg para una teoria de la investigación}

$E_{g}$ interesante que las elaboraciones verdaderas del principio de Goteborg sólo hayan aparecido en el entretiempo ${ }^{5}$ y reflejen un acercamiento tentativo al principio hermenéutico-dialéctico $(H . D .)^{6}$ La teoría goteburguesa de la investigación

1 Con respecto a la crítica y autocrítica de la "teoría crítica" vẻase Böhler, "Kritische Theorie -kritisch reflektiert", en $A R S P$, LVI/4, 1970, pp. 51 ss. Cf. los parágrafos correspondientes en Böhler, Metakritik der Marxschen Ideologiekritik, Frankfurt am Main, 1971. Habermas, Technik und Wissenschaft als 'Ideologie', Frankfurt am Main, 1968, pp. 48 ss. Lobkowicz, "Interesse und Objektivität, en Philos. Rundschau, año 16, diciembre de 1969, pp. 248 ss, Theunissen, Gesellschaft und Geschichte, Berlín, 1969. Wellmer, Kritische Gesellschaftstheorie und Positivismus, Frankfurt am Main, 1969.

2 A diferencia del procedimiento de Kuhn, el método de Goteborg es concretizado por estudios follow-up de procesos de investigación actuales y casos clínicos de las empresas históricas de investigación; ambos son guiados por modelos teóricos de la formación del saber. (Cf. también el trabajo de Elzingas, nota 8.) Apenas puede decirse que Kuhn desarrolle una teoría del progreso del conocimiento. Lakatos va, con sus categorías de la "negative and positive heuristics, regressive and progressive problems shifts", mucho más allá. Pero tampoco él ha desarrollado modelos sobre la situación de la investigación y de los distintos tipos de la formación del saber - como lo hace, por ejemplo, en FIS (cf. nota 3). Cf. el informe sobre las actividades del Instituto en Zeitschrift f. Allgem. Wissenschaftstheorie, II/1, 1971.

3 Radnitzky y Törnebohm, "Forschung als innovatives System", en Zeitschr. f. Allgem. Wissenschaftstheorie, II/2, 1971 (próximamente, FIS).

4 Cf. Radnitzky y Andersson, "Wissenschaftspolitik und Organisationsformen der Forschung", introducción a A. M. Wienberg, Probleme der Grossforschung, Frankfurt am Main, 1970. Cf. además, $P D F$, entre otros, nota 6 .

5 Por ejemplo, Törnebohm, "Science of Science. Lectures at EURATOM", reimp, en J. of Technology and Society, 1971; Radnitzky, "Der Precisbezug der Forschung" (Conferencia con motivo del décimo aniversario del VDM), Studium Generale, 23: 817-855, 1971, abreviado de aquí en adelante como PDF; Törnebohm, "Two Studies concerning the Richelson-Morley Experiment", Foundations of Physics, 1: 47-56, 1970; y "A Foundational Study of Einsteins Space-Time Theory", Scientia, 104/667.688, 1969. Radnitzky, "Theorienpluralismus - Theorienmonismus", en König y Rotter (eds.), Studien zur Wissenschaftstheorie, tomo 6, Messenheim, A. Hein Verlag, 1971, abreviado TP. Törnebohm, "Reflections on Scientific Research", Scientia, 106, 1971; Radnitzky, "Zur Rolle del Fachsprachen in der Produktion von wissenschaftlichen Wissen", en Landgrebe (ed.), Dissenschaft und Philosophie, Akten des II. Deutschen Kongresses für Philosophie, 1971, en impresión. Elzingas, "On Descartes 'Theory of Knowledge and Huygens' Theory of Research", Zeitschr. f. Allgem. Wissenschaftstheorie, II/1, 1971. El trabajo mencionado en la nota $3, F I S$, representa una introducción a la ciencia de la investigación.

6 Cf. en especial, Radnitzky, "Toward a Systematic Study and Criticism of Science and the 
(TI) tematiza la investigación como proceso, en el que no sólo se sistematiza el saber y se produce nuevo saber, sino que también se siguen trabajando los problemas y se crean y mejoran instrumentos - tanto materiales como intelectuales- En el proceso de investigación se interpenetran el saber (S), los problemas (P) y los instrumentos (I), se forman complejos de desarrollo SPI determinados, de tal modo que se habla característicamente en casos marcados de direcciones de la investigación. ${ }^{7}$ A fin de comprender y pronosticar éstas en su lógica, R. quiere iniciar un análisis de los factores generales decisivos, es decir, de las condiciones necesarias de la investigación. Con este planteamiento, la TI demuestra ser por lo pronto una reflexión retrospectiva de la investigación. Sólo cuando se investigan con los resultados esta reflexión (el conjunto de las condiciones, descubiertas como necesarias, de la investigación como investigación) estudios concretos de la investigación y escuelas, puede hablarse en sentido estricto de ciencia de la investigación. R. no ha llegado aún en CSM a esta aclaración de la autocomprensión de su metaciencia como unión entre la reflexión y la ciencia, lo que se expresa también en su posición vacilante frente a la filosofía ${ }^{8}$ y en ocasionales tendencias a reducir la teoría del conocimiento y de la ciencia a ciencia de la ciencia. ${ }^{9}$

La reflexión sobre la investigación que $\mathrm{R}$. ha llevado ya a cabo de facto lo conduce, resumiendo de manera burda, a dos condiciones principales (factors): el preconocimiento (fore-knowledge) del campo de investigación y el sector circundante de la realidad, al que pueden pertenecer "hipótesis" especulativas "sobre la imagen del mundo", y los intereses que guían la investigación, y que con frecuencia se articulan en un ideal de la ciencia. Ambos factores determinan la perspectiva o "modo de ver" y en esa medida son constitutivos del "campo interno de manejo" de una empresa de investigación o de una dirección de investigación. Determinan la perspectiva desde la cual se contempla el territorio (sea como sistema causal, como sistema funcional, etc.). Determinan también la crítica interna y sus criterios. En el caso de la investigación científica, R. cree poder limitar, sin falacia abstractiva, la perspectiva de la TI a este "campo interno de manejo"; no así en el caso de la investigación en las ciencias humanas, ya que aquí el contexto social penetra la investigación concreta, como se demues-

Humanities" (respuesta en un simposio entre autor y crítico de OSH), The Philosophy Forum, 9; sept. de 1971, y Törnebohm y Radnitzky, "Forschung als innovatives System", ya citado, nota 3 .

7 PDF, p. 821.

8 Cf. la crítica de J. Birkhan en Fiener Jahrbuch für Philosophie, tomo II, 1969, pp. $320 \mathrm{~s}$.

- En este contexto resulta especialmente pesada la defectuosa distinción entre los intereses cognoscitivos generales, trascendentales, de los hombres históricos como sujetos cognoscitivos en general, a los que se refiere la reflexión "cognoscitivo antropológica" de Apel y Habermas, y los intereses-motivos especiales, empiricos, ligados a determinadas situaciones históricas (por ejemplo, una dirección de la investigación o incluso de un investigador particular). 
tra con H. D. ${ }^{10}$ A su vez, el "campo interno de manejo" está insertado en diversos "human settings", por los que es influido. A ellos pertenece también el clima intelectual, etc. ${ }^{11}$ Los goteburgueses llevan a cabo el análisis detallado, típico-ideal, de "campo interno de manejo" por medios teórico sistemáticos. Y aquí la teoría reflexiva de la investigación pasa a ser una ciencia de la investigación teórico sistemática, tipificante y "descriptiva".

Los problemas, y el trabajo de investigación que tiende a su solución, son separados típica-idealmente en empíricos (en sentido estricto) y teóricos. A esto corres. ponde la división del trabajo en campos altamente desarrollados de la investiga. ción como la física. Los desarrollos empíricos y teóricos, tanto de saber como de problemas e instrumentos, están unidos por un flujo de información que me. diatiza los problemas. Los empíricos (por ejemplo, los físicos de laboratorio) producen conocimientos aislados, hipótesis de leyes, "encontrados" por el camino empírico. Plantean a los teóricos (los físicos teóricos, etc.) la tarea de situar estos conocimientos aislados dentro del saber previo de las teorías fisicalistas. Si se logra esta asimilación, de tal modo que la hipótesis de leyes pueda deducirse del sistema existente del saber, sólo se muestra que la hipótesis de leyes no contenía un nuevo saber. ${ }^{12}$ Pero'si la hipótesis de leyes no puede ser asimilada, esto puede dar pie a elaborar el sistema de saber pertinente (la teoría fisicalista). Si tampoco se logra esto, el conocimiento aislado sigue siendo un residuo inseguro del saber existente. Ahora bien, un residuo puede crecer, de tal modo que se forme un grupo de conocimientos aislados (por ejemplo, la espectroscopia empírica), que, en ciertas condiciones, llega a entrar en conflicto con teorías establecidas. Con frecuencia esto da pie para un cambio de dirección, algunas veces a un cambio de perspectiva, con lo que surgen nuevos tipos de teorías y hasta se pueden descubrir nuevos campos de investigación. ${ }^{13}$ Por otra parte, los empíricos obtienen hipótesis de los teóricos, hipótesis que han sido derivadas de los modelos teóricos, a fin de que ellos las comprueben. En la física, los empíricos necesitan, a fin de poder ganar datos, sistemas técnicos productores de datos. Para desarrollar éstos, necesitan, desde luego, teorías fisicalistas. Por ello consideran las teorías, desde su punto de vista, primariamente como instrumentos. Esta comprensión instrumentalista de las teorías oculta por supuesto el valor trascendental de posición que las teorias tienen, como formas de descripción, para el empírico. Punto crítico que ha escapado al principio de Goteborg porque no interrumpe la "descripción" teórico sistemática con la reflexión.

$10 P D F$, p. 821 ss. FIS, 102.

11 Esto se expone especialmente en PDF.

12 CSM, I: 185 ss. Cf. el retorno de este argumento en la crítica al empirismo lógico (EL), véase infra. CSM tiene, al final del capítulo sobre aclaración y confirmación, un pequeño párrafo sobre el tratamiento de la problemática en la ciencia de la investigación; este tratamiento es expuesto con más detalle en trabajos posteriores, en especial, FIS.

13 Por ejemplo, TP y FIS. 
A diferencia de los empíricos, aparecen los teóricos, en el modelo teórico sistemático de Goteborg, como representantes de una comprensión reflexiva de la teoría: consideran las teorías primariamente como "naipes" que "copian", estilizándolos, los aspectos escogidos de un campo de objetos. ${ }^{14}$

En tanto no se presente un conflicto de teorias, y sólo se modifique lentamente la perspectiva dominante con el conocimiento y los intereses básicos, tenemos ante nosotros un periodo de "normal science"; la investigación sigue atada a una dirección o a una "escuela", cuyo desarrollo sigue por lo común un esquema del ciclo vital..$^{15}$ Este esquema define la fase final de vejez y esterilidad de una escuela por el paso de una innovatividad autocrítica y referida a lo externo, a la funciona. lidad de un sistema referido a lo interno. Porque la investigación es comprendida, por definición, como sistema innovativo al que no puede hacer justicia ninguna teoría funcionalista de sistemas adaptivos (como la teoría del sistema biológico y cibernético), sino sólo una teoría histórico pragmática de sistemas abiertos e innovativos.

Por consiguiente, el interés particular de los goteburgueses estriba en las fases de partida críticas extremadamente innovativas en las cuales, según los conflictos teóricos, se realiza un cambio de perspectiva y un rompimiento con la tradición -así, pues, en situaciones revolucionarias en las que se ha hecho abruptamente válida la tarea crítica de la tradición hermenéutica, los supuestos ocultos (preconocimiento - en especial imagen del mundo, intereses de la investigación: ideal científico). Tarea que, pasada por alto, se acomete ahora bajo el imperativo de lograr nuevos puntos de vista (; adecuados a la experiencia!) que sirvan de guía.

Los goteburgueses dan un gran significado como factor de manejo a la imagen del mundo presupuesta (con frecuencia tácita, hasta inconsciente o negada en cierta medida). Entre el preconocimiento al nivel de la imagen del mundo (en las ciencias humanas sobre todo la imagen del hombre), y las teorías fisicalistas (o sociológicas, hermenéuticas, social filosóficas) existe un "lazo de acoplamiento retrospectivo". La imagen del mundo se modifica en las crisis, mientras "dirige" la investigación en las fases de la normal science, a saber, como innovación, obstáculo o aun inmunización. En virtud de este efecto direccional, empíricamente calculable, sobre todo del presupuesto de formación del mundo, pueden presentarse prognosis sobre el desarrollo de una investigación o de direcciones completas de la investigación.

Por la pretensión a prognosis condicionadas, colide TI con la crítica del historicismo global de Popper. Si bien son de origen popperiano y ven como logro de Popper el haber devuelto en parte la dimensión histórico pragmática a la teoría de la ciencia, en contra de los positivistas lógicos, los goteburgueses deben pre-

14 PDF, p. 819. El contexto dice así: "Un saber determinado trae a la conciencia un no saber muy determinado. En el $\mathrm{S}$ se forma asi un círculo de preguntas, de problemas P".

15 CSM V: XXXIII ss., esboza este esquema del ciclo vital de una tradición de investigación, que se expone en "lenguajes técnicos" (cf. supra nota 6). 
sentar un frente ante Popper. La lógica del problema y su interés práctico ${ }^{16}$ en el control de la fuerza liberada de productividad de la ciencia por el análisis de sus "leyes de movimiento" y por un planeamiento racional de la ciencia, los empuja hacia la cercanía de la tradición marxista y su ampliación crítico filosófico tras. cendental (Habermas, Apel, entre otros). ${ }^{17}$

R. reprocha a Popper el manejar las conjeturas como blackbox. Por el contrario, la TI quiere descubrir aquí regularidades en la secuencia de problemas, en las estrategias, etc. Quiere demostrar que no existen "malas infinitudes" de alternativas posibles, sino que éstas están estructuradas siempre ya como un árbol limitado de decisiones y que con cada rasgo ulterior pueden ser delimitadas más estrechamente. Ahora bien, el aspecto del rasgo siguiente no está dentro del arbitrio del teórico que planea libremente, sino que depende en primer lugar del terreno de la investigación, en segundo de las reglas de juego del actuar científico según metas racionales, y en tercero de los factores de manejo no problematizados, y que por ello obran casi causalmente como la imagen del mundo y el ideal de la ciencia.

De acuerdo con la base goteburguesa, las tendencias de prognosis son, desde luego, posibles, lo que sería una suposición de la racionalización premeditada de la politica de la ciencia. En ello estriba la significación práctica de este principio. En qué medida logre en general este principio liberar a los investigadores de su autocomprensión cientista, que no toma en consideración, en especial como consecuencia de EL, la verdadera mediación por la dimensión histórico prgmática, sigue siendo dudoso, en tanto que el principio mismo de Goteborg descuide la reflexión acerca de esta dimensión y su inclusión en ésta a favor de la objetivación y "descripción" teórico sistemática. Las últimas declaraciones van tentando el camino de una comprensión de la TI como "ciencia hermenéutica del logro cognoscitivo en los procesos de investigación", pero aún no se ve muy bien en qué medida se hayan sacado las conclusiones de ello.

¿Qué hace en realidad la TI? Desarrolla un marco de interpretación general del actuar científico, en el que entra lo típico de este actuar, de tal manera que el marco sea transferible a la investigación histórica, presente y futura (en primer lugar de las ciencias naturales). Una vez que se hayan incorporado a él las condiciones específicas individualizadas, deberá hacer incomprensible, para nosotros y para los investigadores, sobre todo el desarrollo de una empresa de investigación, una escuela o toda una dirección de la investigación por medio de cuasi aclaraciones teórico sistemáticas de la prognosis anterior y condicionada del curso posterior. Lo que $R$. destaca del paradigma del psicoanálisis de Apel-Habermas. Ricceur, la obtención de la aclaración por la comprensión, caracteriza al método -que interroga analíticamente la autocomprensión del investigador- como her. menéutico, crítico de la tradición, de la TI misma. Cuenta, en la misma medida que la crítica marxista de la ideología, con relaciones externas entre la autocom-

16 CSM II, 118.

17 CSM, II, 114 ss., en especial 118 ss. 
presión oficial y el proceso fáctico de investigación, es decir, con una disensión inconsciente entre "teoría y praxis" (Marx), entre "juego de lenguaje y forma de vida" (Wittgenstein). Por ello a fin de hacer clara la relación debe "a-clararla", tomando en consideración una cuasi causa, en lo que la causa no es material, como en la crítica marxista, sino que es inherente a la mediación no comprendida y teórica de la tradición. Aquí la cuasi causa es justo un ideal de la ciencia o imagen del mundo no hermenéuticamente explicado y que por ello cumple con una función de manejo. ${ }^{18}$ Precisamente este efecto cuasi causal de tales tacit presuppositions hace posible las prognosis condicionadas, cuyo status no concuerda, desde luego, con el de las teorías generales (por ejemplo, de las ciencias naturales), sino que está ligado a la lógica específica de interpretaciones generales que Habermas ha desplegado con el ejemplo del psicoanálisis. ${ }^{19}$ En suma, los problemas teórico científicos de la TI goterburguesa están justamente en el nivel que refleja el principio hermenéutico dialéctico en los ejemplos de la crítica de la ideología y la hermenéutica, el modelo de psicoanálisis, la aclaración histórica, etc. En esa medida debería el HD, como teoría especial de la ciencia de las ciencias humanas, que como tal lo honra R., dar el marco teórico científico para la TI.

\section{El postulado de una politica critica de la ciencia. Propuestas de solución al problema fundamental de la política de la ciencia}

En realidad, la expansión del principio de Goteburgo debería ir seguida por la del $\mathrm{HD}$, a fin de interrogar después a ambas con respecto a su aporte a la solución del problema que consideramos como fundamental en una política de la ciencia y que vemos aun quizá como el problema teórico y práctico de la humanidad actual: el conocimiento y la dirección responsable del progreso cientifico y técnico. Con todo, presuponemos aquí en lo esencial el principio hermenéutico dialéctico y sólo lo discutimos en la medida en que nos parece necesario para la aclaración de su aporte general a la solución del problema inicial $y$ en especial para la aclaración de la autocomprensión de una investigación social crítica y una po. lítica científica.

18 Al subrayar Radnitzky el valor de la TI para los problemas social científicos de las fases cuasi naturalistas del actuar social, presupone justo este proceder de la cuasi aclaración. Pero esto significaría para la TI que no podría comprenderse simplemente como teoría de sistemas, sino que deberia entenderse como ciencia humana hermenéutica y crítica de la tradición, que puede trabajar, con respecto a las fases cuasi naturalistas del proceso de investigación (jque deberían ser superadas en la conciencia del investigador por una aclaración del tipo de la TI), con los medios de la teoría funcional analítica de sistemas. En tanto no se ponga en claro esto, no parece infundada la suposición de que la TI no está libre de la fallacy cientista naturalista y de que su autocompresión está -injustificadamente-- orientada al objeto (naturaleza) de su campo de objetos (la formación del saber en las ciencias naturales). Cf. en tanto John 0'Malley, "The Dialectic of Inquiry", en The Philosophical Forum 9, núm., 4, 1971. O'Malley aboga por la inclusión de la TI en una dialectically conceived semiology.

19 J. Habermas, Erkenntnis und Interesse, Frankfurt am Main, 1968, en especial, pp. 324 ss. 
La respuesta a la pregunta de dentro de qué marco ha de solucionarse ese problema, será esbozada por nosotros, con respecto a la TI y HD, en cuatro puntos:

1) Una ampliación del modelo psicoanalítico puede proporcionar el marco para una toma de dirección realmente democrática y una posible superación de los resultados colaterales socio culturales del progreso técnico científico, dirigido hasta ahora ni "crítica", ni "autoconsciente" y ni "solidariamente" (en el sentido de la tradición marxista), sino de modo relativamente autónomo y dentro del marco de intereses capitalistas particulares. Pensamos en resultados colaterales como el aumento de la desigualdad social, como la acumulación de la miseria material y sobre todo la explotación a nivel mundial del proletariado (Tercer Mundo) y en resultados colaterales como la presión ideológica y la "tolerancia represiva" (Marcuse).

La postulada ampliación del modelo psicoanalitico se refiere al aspecto, que la investigación social crítica ha hecho válido, de la emancipación material de la miseria $y$ la desigualdad social. Meta que sacude tanto antes como después las relaciones básicas de las sociedades capitalistas - y estatal capitalistas. En contra de ello, la mera investigación social empírica, que racionaliza dentro del sistema de valores sociales previamente encontrado sólo la elección media, sirve única. mente para el mantenimiento del status quo. ${ }^{20}$ La oculta filosofía de la historia de la limitación a los medios, que depende del principio empírico de la neutralidad de los valores, se ajusta demasiado bien al marco de las sociedades que han avan. zado técnicamente por el camino de la "tecnocracia". Por el contrario, la "investigación social crítica" depende - por su meta de emancipación sustancial- de un enlace con una filosofía de la historia "planeadora": debe construir teorias del desarrollo social que muestren en qué medida son eliminables la desigualdad social, etc., y qué proyecto alternativo es objetivamente prometedor. ${ }^{21}$

La perspectiva de una investigación social crítica dirigida _en el sentido de la tradición marxista- sobre todo a la emancipación material señala un deside. ratum decisivo en el modelo psicoanalítico. ${ }^{22}$ Por otra parte, esto no limita la pretensión de validez de aquél en sentido teórico científico y metodológico; pues también la investigación social crítica, y justamente ella, depende de la mediación de "comprender" y "aclarar" de acuerdo con el ejemplo de un psicoanálisis bien entendido. ${ }^{23}$ Ahora bien, ¿cómo podría ampliarse este modelo también en sentido práctico de modo que - visto político económicamente- represente más de una sola perspectiva liberal, y pueda prestar servicio a una mediación emancipadora teoría-práctica ( en el sentido de la tradición marxista!)? Esbocemos muy brevemente la respuesta.

20 Así también Radnitzky, Contemporary Schools..., $2^{\text {a }}$ ed., Goteborg y New York, 1970 (próximamente $C S M$ ), tomo II, pp. 152 ss.

21 CSM II: 147 ss.

22 También McLaughlin en Telos.

23 Cf. Apel, "Szientistik, Hermeneutik, Ideologiekritik", Jahrbuch, I, 1968, cap. II, loc. cit. supra. Cf. Habermas en Continuum, vol. 8, núms. 1-2, en especial, p. 128. 
Las condiciones materiales y la desigualdad política práctica (como la explotación y el dominio de clase) son barreras a la comunicación y la autorreflexión. Esto puede mostrarlo la ideología crítica del tipo de la crítica formal que, como hermenéutica profunda, sólo descubre perturbaciones de la comunicación por caminos analítico normativos y con pretensión a la validez intersubjetiva. ${ }^{24}$

Desde luego, tal crítica ideológica es relativizada por problemas hermenéuticos de interpretación, donde y en la medida en que su "análisis" depende de la construcción de imágenes situacionales de gran extensión o que abarquen la sociedad completa.

En todo caso, puede presuponer el carácter normativo de sus puntos de vista directivos y contrafácticos como intersubjetivamente obligatorios. La comunicación y la autorreflexión son, a saber, junto con la autoconservación, las condiciones de posibilidad del actuar humano, es decir, con sentido, y posiblemente consciente en su más amplio sentido. Como factores "trascendentales" necesarios para un actuar mediatizador de conciencia y de sistemas sociales de actuación, que en tanto mediatizadores de conciencia son siempre ya "sistemas culturales de interpretación" (Habermas), pueden ser señalados como generalmente válidos por teorías de la linea de la reflexión (antropología del conocimiento, teoría de la competencia co. municativa, teoría trascendental de lo social).$^{25}$

Ahora bien, si, como suponen el funcionalismo y la teoría del sistema, es válido el problema de la conservación para el metaproblema $y$, con ello, para la orientación de la actuación de la planeación social $-\mathrm{y}$ esto significa en medida cada vez mayor la política de la ciencia-, entonces habría que hablar también del riesgo de los sistemas sociales de actuación e interpretación en un marco mayor: también se correría el riesgo de la reducción de la comunicación y de la posible emancipación por medio de la autorreflexión critica. ${ }^{26}$

El resultado indicado de la reflexión filosófica pondría en manos de la polí. tica de la ciencia los criterios racionales generales para guiar el progreso cientí. fico, es decir, para la crítica de su anterior crecimiento natural o para su futura dirección tecnocrática. Con ello, quedaría establecida al mismo tiempo la política de la ciencia sobre el tipo de una mediación emancipadora teoría-praxis, tipo fundado sobre aquella reflexión $y$ sobre la prueba crítico ideológica de una limitación represiva de la comunicación y autorreflexión, y sobre la propuesta metacrí.

3.4 Con respecto a esta diferenciación de tipos de crítica, ef., en primer lugar, Böhler, Metakritik..., pp. 42 s.; cf. pp. 46 s., en especial la nota.

25 Cf. el citado ensayo de Apel; cf. Habermas en Habermas-Luhmann, Theorie der Gesellschaft..., Frankfurt am Main, 1971. Cf. Böhler, "Rechtstheorie als kritische Reflexion" (en especial pp. 62-97), en Rechtstheorie, ed. G. Jahr y W. Maihofer, Frankfurt am Main, 1971. Además Apel, "Szientismus oder transzendentale Hermeneutik?" en Hermeneutik und Dialektik, tomo I, ed. R. Bubner y otros, Tubingen, 1970; Apel, "Die Kommunikationsgemeinschaft als Transzendentale Vorausetzung der Socialwissenschaften", aparecerá en Acta Soziologica, 1971.

${ }^{26}$ Cf. Böhler, loc. cit., en especial p. 93. 
tico socialista que exige un cambio de sistemas; a saber, sobre el "principio regulativo" de realizar siempre, en la mayor cantidad posible, la comunicación pública y autorreflexión.

La tarea de una mediación teoría-praxis y de la política de la ciencia entendida en este sentido, estriba en suprimir el mencionado riesgo de la sociedad, dirigiendo por medio de "esbozos" contrafácticos y proposiciones de acción una praxis que modifique la sociedad fáctica en una "forma cercana" a una libre sociedad de comunicación, posible en ciertas condiciones. ${ }^{27} \mathrm{El}$ establecimiento de los cambios de la esfera institucional y productiva, necesarios para tal sociedad de transición, no puede llevarse a cabo, si es que quiere permanecer dentro del marco de la racionalidad científica y/o del marco de los intereses emancipadores, ${ }^{28}$ ni en forma técnico social ni por una "revolución desde arriba". Dado que el cambio que se propone el socialismo debe emprenderse a partir de modelos alter. nativos sociales, y éstos contienen momentos comprometidos de la interpretación de la situación y del "esbozo" alternativo, orientados hacia valores, el cambio socialista está obligado a una comunicación autocrítica en la sociedad. Esto quiere decir, empero, que también el acto material del cambio emancipador exige, si no quiere sacrificar su carácter racional y con él su carácter emancipador, la mediación por comprensión, desplegada a partir del modelo del psicoanálisis. Así queda fundamentado sistemáticamente, para la práctica socialista, lo que la oposición izquierdista en el socialismo (por ejemplo, Rosa Luxemburgo, Kronstadt, Alessandra Kollontai y la oposición de los trabajadores soviéticos en 1921-22, Posen y Hungría en 1956, Kolakovski, Bloch, Harich, Havemann, Praga en 1968) combatió desde mucho en forma comprometida: el proceso revolucionario de cambio no depende de una anticipación práctica (creciente) de la opinión pública democrática (sociedad de comunicación) ${ }^{20}$ a cuya creación tiende, sin embargo, en sentido amplio, su interés emancipador. Así se comprueba la figura de la "espiral hermenéutica" como estructura de una mediación emancipadora teoría. praxis. ${ }^{30}$

2) A fin de que la mencionada mediación emancipadora teoría-praxis, a cuyo servicio estaría la crítica de la ideología y la investigación social crítica como ciencias guías, no equivoque las causas concretas de esos efectos colaterales, que yacen en el entrecruzamiento de la investigación con el complejo de intereses

27 Habermas, loc. cit., y Böhler, Das Problem des 'emanzipatorischen Interesses' und seiner gesellschaftlichen HFahrnehmung, cap. III, en especial pp. 238 ss., en ZEE, año 14, 1970, p. 47 (en Man and World, II, 1970).

28 Con respecto a la relación entre la racionalidad social científica y el momento emancipador de los intereses cognoscitivos hermenéutico-prácticos, de los que aquí se trata particularmente, cuando se habla de 'interés emancipador', véase Böhler, loc. cit., en la nota 25, pp. 108-113.

${ }^{29}$ Cf. finalmente A. Wellmer, véase supra nota 1, J. Perels; "Zur politischen Verfassung des Sozialismus", en Kritische Justiz. Böhler, Metakritik, 1971, 2.

${ }^{30}$ Cf. Böhler, loc. cit. del op. cit., en la nota 27 , cap. III. 
industriales, militares y políticos, debe establecerse, una investigación social crítica justo para este campo de interdependencia. ${ }^{31}$ Pero precisamente esto faltó hasta ahora. ${ }^{32}$

Aquí habría que discutir si puede comprenderse el funcionamiento natural de este complejo dentro del marco de un análisis del capitalismo y con los medios de un análisis historizado, a saber, crítico ideológico, de las funciones, que acepta como problema de referencia la supervivencia del sistema y parte de un valor histórico descriptivo de la tesis de la tecnocracia. Esta proposición, hecha por Offe, tuvo como consecuencia que los actuales sistemas capitalistas resuelvan su problema de supervivencia y estabilización en medio de una creciente destrucción de las instituciones de la opinión pública burguesa, mediante la defensa (técnico social) de los "riesgos técnicos", que se producen por si solos, y mediante la represión o canalización permanente de las crisis que surgen. ${ }^{33}$

Ahora puede concretarse el problema de referencia manifiestamente por un enlace con la autocomprensión y la orientación de la acción confesada o programática del sistema económico político. Tal enlace hermenéutico y crítico ideológico es posible, ya que tenemos que ver en las naciones industrializadas de Occidente (organizadas capitalistamente) y de Oriente (directamente estatal capitalistas) con sistemas en los que interviene el Estado o en los que dirige. Aquí no media ya ese abismo contradictorio entre la proposición político social de una meta y el curso fáctico del sistema, que todavía Marx presupuso al identificar la política, (derecho, etc.) con la ideología. En mi opinión, esto se muestra, con bastante claridad, en la búsqueda de las causas del envenenamiento de la ecósfera, que tropieza con una praxis, determinada por el fetiche del "crecimiento económico". Así, por ejemplo, en la República Federal Alemana una de las metas oficiales de la política económica es el crecimiento económico junto con la estabilidad; desde luego, de facto funciona como la meta del sistema político económico.

3) El envenenamiento del ambiente convertido así en problema existencial, debido a tal determinación de metas, podría provocar una reorientación parcial del sistema económico político. Un interés vital general se enfrenta al interés del sistema. De modo típico, sólo llega a tener la fuerza de la confrontación y el carácter de apremiabilidad en la crisis. Hasta ese momento, el sistema suprime o reprime los intereses prácticos vitales por mor de los intereses del sistema (en la medida en

31 Cf. Rainer Rilling, "Forschungspolitik der BRD", en A. N. Weinberg, Probleme der Grossforschung, Frankfurt am Main, 1970, pp. 275 ss. Cf. Radnitzky y Andersson, 'Wissenschaftspolitik", ibid., pp. 9 ss. Cf. H. Krauch y otros, Forschungsplannung, München, 1966, y Die organizierte Forschung, Neuwied-Berlín, 1970; Cf. D. Senghaas (ed.), Friedensforschung und Gesellschaftskritik, München, 1970.

32 Cf. C. Grossner, en Zeit, núm. 12, 1971, pp. 46 ss.

33 Cf. Offe, "Das politische Dilemma der Technokratie", en Text zur Technokratiediskussion, ed. C. Koch y D. Senghaas, Neuwied-Berlín, 1970; "Politische Herrschaft und Klassenstrukturen. Zur Analyse spätkapitalistischer Gesellschaftssysteme", en Politikwissenschaft, ed. G. Kress y D. Senghaas, Franfurt am Main, 1969, y Der Mensch in der geplanten Welt, ed E. Kramm, Stuttgart, 1970. 
que aquellos no son fomentables). El ejemplo del envenenamiento ambiental sólo manifiesta así la reducción de la comunicación y la autorreflexión en el sistema. En esa medida, los efectos colaterales, que se han hecho visibles en el problema del envenenamiento ambiental del progreso científico-técnico crecido de modo natural, señalan a su mediación en la estructura política y económica del sistema, cuya represividad es responsable de los mencionados efectos colaterales político culturales (véanse pp. 275 y 282 ).

Así, pues, con respecto a la política de la ciencia, pueden producirse, a partir del problema del envenenamiento del ambiente, una concretización de su "principio regulativo", en la medida en que se señala aquí un posible lugar de ruptura en el mecanicismo funcional, sobre todo, quizá, en los sistemas occidentales aún no estatalmente dirigidos. A fin de percibir esta posibilidad, la política de la ciencia debería evitar dos posibilidades de solución de la crisis ecológica, que se muestran como peligros a partir de la perspectiva de la reflexión y la critica de la ideología. Por una parte, la posibilidad dirigida estatalmente de solucionar la crisis ambiental, administrativamente desde arriba: sin modificación de sus instituciones, que ofrecen las posibilidades de despliegue concretas para la vida pública y representan pasos en el camino de una 'superación' realmente democrática del sistema. A esto se agrega el peligro que representan las medidas prácticas para la solución del problema ambiental en los países capitalistas al tender al establecimiento de una industria ambiental, que fortalece tanto el poder intervencionista del Estado, como cimenta también (y sobre todo) las condiciones capitalistas de producción. Ambos efectos no coliden por ello entre sí, más bien están en armonía uno con otro y, a saber, tanto más mientras más consecuente sea la transición de las condiciones de competencia de la economía privada hacia un sistema de crecimiento y equilibrio de economía total, que es dirigido de acuerdo con el interés del capital por el gobierno. ${ }^{34}$ Esta dirección conlleva, en la República Federal Alemana, una destrucción de la estructura federativa y una "desdemocratización de los procesos de decisión en el terreno de la constitución financiera" $;^{35}$ tendencias que sólo resultan fortalecidas por el establecimiento de una industria ambiental de acuerdo con el ejemplo sueco. ${ }^{36}$

De los peligros ya mencionados, resultan las siguientes tareas para la politica de la ciencia.

3.1. Es de esperarse que la industria ambiental deba ser organizada en forma que tenga utilidad común e independientemente de la federación y de empresas económicas. Sería pensable también la forma de una institución de derecho público.

3.2. Dado que en los nuevos productos y tecnologías de la investigación (I) y el desarrollo (D), resulta con frecuencia imprevisible o insuficientemente impre-

34 Cf. J. Huffschmied, Die Politik des Kapitals, Frankfurt am Main, 1971.

${ }^{35}$ Loc. cit., pp. 178 ss.

36 Cf. G. Graffenberger, "Mit Umweltschutz lässt sich auch Geld verdienen", Frankf. Rundschau. 
visible el espectro de los posibles resultados negativos y los peligros ecológicos in statu nascendi, dependería de la institucionalización de una investigación de efectos y anticipación científica de los mismos, simultánea a la investigación (I) y el desarrollo (D), es decir, de una futurología en un sentido concreto, que debería estar bajo la protección de una legislación correspondiente, antes de poder colocar tecnologías y productos nuevos. Andersson y Radnitzky lo señalan en su introducción crítica a las Reflexions on Big Science de Weinberg. ${ }^{37}$

3.2.1. Estos institutos deberían ser independientes tanto del Estado como de la industria. Deberían tener el status de insituciones de derecho público y una "sección de conexión retrospectiva" para la comunicación con la opinión pública. ${ }^{38}$

4) Junto con la tarea de vencer los efectos imprevistos del progreso técnicocientífico, recaería en una política crítica de la ciencia, concebida en el sentido de una mediación emancipadora teoría-praxis, la tarea principal de tener bajo control también a las leyes del movimiento (Marx) de la fuerza productiva de la ciencia misma. Es posible, según Andersson y Radnitzky que se trate alli de una tarea de planeación "interna" teórica y a la vez "externa".

4.1. El desarrollo del proceso científico y de investigación mismo figuraba como "cosa en sí" o blackbox. Ahora bien, la investigación de la fuerza productiva 'ciencia' misma en sus regularidades sería justo la tarea específica de una ciencia de la investigación tal como la desarrolla la escuela de Törnebohm. Como se expuso en el parágrafo I, vemos este principio como el marco con posibilidad de desarrollo para la institucionalización de una autorreflexión específica del investigador. ${ }^{39}$ (Desde luego, abogaríamos, con Radnitzky y Andersson, por su complementación por medio de la reflexión general teórico científica y filosófica del HD).

4.2. Sólo nos es posible adoptar una posición frente a la tarea planificada bajo unos cuantos aspectos, que entenderíamos en el sentido de los lemas "de ser posible mucha transparencia y democracia en el 'campo de manejo externo' de la investigación y protección del 'campo de manejo interno' frente a influencias deformadoras por parte del 'externo" ".

La tarea que acabamos de mencionar la ha fundamentado suficientemente Radnitzky. ${ }^{40}$ La autonomía de la investigación de los fundamentos, postulada por él y por Andersson, plantea desde luego el problema de la repartición de los medios y de las prioridades. ${ }^{41}$ Tendría que discutirse la pregunta de las instancias pertinentes o que habría que crear. Por el contrario, de la concretización crítico ideo-

37 Wissenschaftspolitik..., p. 21.

38 Cf. R. Jungk, "Vorschlag zur Errichtung eines europäischen Lookon-Instituts", publicado en $A R C H, 1968,1$, p. 30 . Tal división habría de tener también en un instituto de investiga. ción ecológica la doble tarea de informar sobre el trabajo del instituto y sobre las proposiciones, observaciones, etc., que se presentan al instituto.

39 C. $P D F, \S 41$.

40 Ibid., § 32, pp. 834 ss., y Wissenschaftspolitik..., 48 ss., 18, cf. § 3.

41 Wissenschaftspolitik..., p. 41. 
lógico de los principios de la comunicación y la autorreflexión, podría surgir en qué dirección habría que buscar la respuesta: a saber, en el sentido de una independencia del Estado y la industria. Esto correspondería también al principio de la autoconservación. ${ }^{42}$ A fin de no depender de la industria y convertir sus funciones en tecnología, la investigación está abocada a medios estatales, que podrían ser completados por aportaciones industriales forzosas ('impuesto para la investigación'). Pero ¿cómo podrá asegurarse así la independencia frente al Estado? ¿Acaso por la institución de un consejo de investigación que se formaría de miembros escogidos de las instituciones de investigación, de la vida pública (¿cuál?, ¿cómo se eligirían?) y de los partidos? ¿Cuál de las soluciones hace posible, al lado de la autonomía, una mayor transparencia y democracia dentro de un sistema crecientemente integral y no democrático, que no tiende a la transparencia?

¿Sería el medio adecuado reintegrar la investigación de los fundamentos a la universidad? Sin duda alguna, debido a la transparencia allí más fácilmente institucionalizable y, en el caso de las humanidades, por una razón sistemática: están destinadas, por principio, a la comunicación; aquí investigación y 'enseñanza' no son separables. ${ }^{43}$ Pero también en el caso de la investigación científica de los fundamentos son necesarias, por lo que respecta al marco teórico y los problemas relacionales que lo constituyen (ideal de la ciencia, imagen de mundo), la crítica de la tradición, la comunicación crítica y su concretización mediante "la competencia entre las direcciones de la investigación", tal como lo muestra la TI goteburguesa. ${ }^{44} \mathrm{~A}$ pesar de las tendencias de la formación escolar podría realizarse la necesaria organización interna de la investigación de los fundamentos como "sistema inorativo" en determinaciones adecuadas de escuelas de altos estudios, dentro del marco de la universidad, sobre todo cuando las direcciones particulares de las materias se enlazan sistemáticamente con la teoría y la filosofía de la ciencia. ${ }^{45}$

Esta solución es contradicha por los argumentos realistas de $S c h e l s k y,{ }^{46}$ y no en úlimo término por la división entre formación e investigación. Pero el modelo Bielefeld de Schelsky, sobre todo la integración del ZIF en la universidad de Bielefeld, ¿no muestra acaso una posible solución de la contradicción entre reintegración y "emigración de la investigación"? Quizá debieran concebirse y realizarse escuelas conjuntas de altos estudios con divisiones de subgraduados y graduados. Las primeras se concentrarían en la formación, que, sin embargo, tendria que ampliarse por un estudio fundamental obligatorio político social y de teoría de la

42 Ibid., p. 49.

43 Cf. Böhler, "Zur Idee der kritischen Universität", Feature NDR, III, 9 de enero de 1969. Cf. Apel, "Wissenschaft oder Emazipation? Zeitschr. f. Allgem. Wissenschaftstheorie, 1970, 1/2. Cf. Böhler, Entwurf einer Hochschule im Sinne der Theorie-Praxis.Vermittlung, manuscrito inédito, 1970.

44 Véase supra, cap. I: cf. también PDF, \& 41.

45 Así Böhler, Entwurf...

${ }^{46}$ H. Schelsky, Abschied von der Universitätspolitik, Gütersloh, 1969. 
ciencia, lo mismo que por estudios de proyectos interdisciplinarios, lo que evita la reducción a la idiocia monotemática y la tecnología.

Las últimas tendrían que subdividirse a su vez en menor medida, en investigación de fundamentos $e$ investigación de proyectos a fin de que la Universidad no perezca por anemia de "fragmentation and purity of science". ${ }^{4 \tau}$

Desde luego, la investigación de proyectos exige en gran medida, por razones prácticas, institutos propios, y podría tender como suponen con derecho Andersson, Radnitzky y otros, a soluciones europeas. ${ }^{48}$ Sin embargo, sería más problemático garantizar transparencia y publicidad de lo que parecen suponer los optimistas protagonistas de los grandes institutos europeos..$^{49}$

UNIVERSIDAD DE SAARBRÜCKEN

SEMINARIO DE FILOSOFÍA

Dietrich Böhler

(trad. de Elsa Cecilia Frost)

47 Así, Weinberg, según Radnitziky y Andersson, p. 55.

48 Loc. cit., pp. 30 ss.

49 Por ejemplo, R. Jungk, loc. cit., pp. 25 ss. 